\title{
A Test for Perceptual Independence With Dissimilarity Data
}

\author{
Nancy A. Perrin \\ Portland State University \\ F. Gregory Ashby, University of California \\ Santa Barbara
}

\begin{abstract}
The dominance axiom states that the dissimilarity of a pair of stimuli differing on two dimensions must exceed the dissimilarities of the corresponding pairs of stimuli that differ on only one of the dimensions. This axiom is presented as a test of dissimilarity data to determine if the dimensions of the perceptual space are perceived independently, and as a diagnostic tool in assessing the INDSCAL model's assumption of independent dimensions. The general recognition theory of similarity, which contains both the three-mode and INDSCAL multidimensional scaling models as special cases, is used to motivate the test. It is shown that general recognition theory predicts consistent violations of the dominance axiom with dependent
\end{abstract}

\begin{abstract}
dimensions, but not independent dimensions. A consistent pattern of violations of dominance suggests that the three-mode model is most appropriate. When the test of dominance is satisfied, the INDSCAL model is appropriate for the data. A simulation study was conducted to examine the pattern of violations of the dominance axiom when varying degrees of perceptual dependence exist. An examination of dissimilarity data from a study of the size-weight illusion revealed the expected pattern of violations of the dominance axiom. Index terms: dominance axiom, general recognition theory, INDSCAL, MDS, perceptual independence, three-mode model.
\end{abstract}

The selection of a multidimensional scaling (MDS) model is influenced by the model's assumptions about the perceptual space. For example, the Simple Euclidean scaling model (McGee, 1968) assumes that the dimensions are perceived independently by each person and weighted equally across people. The Weighted Euclidean scaling model (or the INDSCAL model; Carroll \& Chang, 1970; Horan, 1969) also assumes that the dimensions are perceived independently by each person; however, differential weighting of the dimensions is allowed by each person, and these weights can vary across people. Finally, the General Euclidean scaling model (or the three-mode model; Carroll \& Wish, 1974; Tucker, 1972) does not assume that people perceive the dimensions independently. This model allows for differential weighting of dimensions by each person, differential degrees of dependency between pairs of dimensions within each person's representation, and it allows the dimension weights and degrees of dependency to vary across people.

There are several three-mode models, and each has unique properties and interpretations (Bloxom, 1978; Carroll \& Chang, 1972; Young \& Lewyckyj, 1979). Because the differences between the various three-mode models are not relevant to the issues examined here, Tucker's framework for threemode MDS was used.

The most important property of the various three-mode models is their ability to define the perceived dissimilarity of stimulus $S_{\mathrm{A}}$ to $S_{\mathrm{B}}$ for person $i$ as

APPLIED PSYCHOLOGICAL MEASUREMENT

Vol. 15, No. 1, March 1991, pp. 79-93

(C) Copyright 1991 Applied Psychological Measurement Inc.

0146-6216/91/010079-15\$2.00 
$d_{i}\left(S_{\mathrm{A}}, S_{\mathrm{B}}\right)=\left[w_{u i}^{2}\left(u_{\mathrm{A}}-u_{\mathrm{B}}\right)^{2}+w_{v i}^{2}\left(v_{\mathrm{A}}-v_{\mathrm{B}}\right)^{2}+2 w_{u i} w_{v i} \cos \left(\theta_{i}\right)\left(u_{\mathrm{A}}-u_{\mathrm{B}}\right)\left(v_{\mathrm{A}}-v_{\mathrm{B}}\right)\right]^{1 / 2}$,

where $\theta_{i}$ is the angle between dimensions $u$ and $v ; w_{u i}$ is, for example, the weight associated with the $u$ dimension for person $i$; and $u_{\mathrm{A}}$ is, for example, the coordinate on the $u$ dimension for stimulus $S_{\mathrm{A}}$. All people are assumed to use the same stimulus coordinates, but the weights for the dimensions and the angle between dimensions are allowed to vary within as well as across people. The INDSCAL model results when the last term of Equation 1 is dropped, and the Simple Euclidean model is a special case of the INDSCAL model in which all dimension weights are equal.

As Equation 1 makes apparent, an important factor to consider when selecting an appropriate MDS model is whether the dimensions of the perceptual space are independent, because the last term in Equation 1 allows for dependent dimensions. It is possible in some cases to transform linearly a space with oblique or dependent dimensions to a space with orthogonal or independent dimensions (MacCallum, 1976b). Therefore, there may exist an INDSCAL model that can provide as good fit as the three-mode model for a given set of data with dependent perceptual dimensions. Because the transformation changes the weights for each dimension, the transformed threemode and INDSCAL solutions may lead to different interpretations of the same data. If the researcher uses the INDSCAL model, the recovered dimensions will not correspond to the true perceptual dimensions.

Many instances can be found in the psychological literature in which physically distinct stimulus components are perceived dependently. With the wavelength and intensity of light, for example, a change in wavelength only will cause a change in hue, but it will also often cause a change in brightness. Another example is the size-weight illusion in which larger objects tend to be judged heavier than smaller objects of the same mass.

The angle $\theta$ in Equation 1 can be used as a measure of perceptual dependence. In fact, Dunn (1976) used the angle between dimensions derived from the three-mode model to investigate individual differences in the strength of the size-weight illusion.

MacCallum (1976a) showed that the INDSCAL model will not necessarily detect violations of the assumption of independent dimensions. He simulated dissimilarity data with oblique dimensions, found good fit of the INDSCAL model to the data, and concluded that violations of the assumption of independent dimensions could go unnoticed with the INDSCAL model and lead to an unreasonable solution in which the recovered dimensions do not equal the true dimensions. He also showed that a good fit of the INDSCAL model does not necessarily imply that the assumption of independent dimensions is satisfied. He suggested looking for negative person weights and correlated stimulus coordinates in an INDSCAL solution as evidence that the assumption of independence is violated. When Dunn (1976) analyzed his size-weight illusion data using INDSCAL, however, he did not find negative person weights or any indication that the INDSCAL model was inappropriate, even though this is a case in which the assumption of independence should be violated. Therefore, a separate test of dissimilarity data is needed to determine if the dimensions are perceived independently.

A diagnostic tool for testing perceptual independence with dissimilarity data is suggested here. The test is based on an axiom called dominance, proposed by Tversky and Gati (1982) as a constraint on a general class of models that they called monotone proximity structures. In addition to providing important information about how people process the stimulus dimensions, the test guides the researcher in selection of the appropriate MDS model. The use of dominance to test independence is motivated by general recognition theory (Ashby, 1988; Ashby \& Perrin, 1988; Ashby \& Townsend, 1986) and its definition of perceptual independence. 


\section{General Recognition Theory}

General recognition theory has been used to examine varieties of perceptual independence (Ashby, 1988; Ashby \& Townsend, 1986), decision rules in the perception and categorization of multidimensional stimuli (Ashby \& Gott, 1988; Ashby \& Maddox, 1990), perceived similarity (Ashby \& Perrin, 1988), and preference judgments (Perrin, 1986). This theory assumes that presentation of the same stimulus does not always produce the same perceptual effect; thus, perceptual effects $x$ and $y$ are random with probability density function $\mathrm{f}(x, y)$. In an identification experiment with the two stimuli, $S_{\mathrm{A}}$ and $S_{\mathrm{B}}$, the person is assumed to divide the perceptual space into regions and to assign a response to each region. For each trial, the person determines in which region the percept is located and then emits the associated response ( $R_{\mathrm{A}}$ or $R_{\mathrm{B}}$ ). The probability of confusing $S_{\mathrm{B}}$ for $S_{\mathrm{A}}$ in the two-dimensional case is given by

$P\left(R_{\mathrm{B}} \mid S_{\mathrm{A}}\right)=\int_{r_{\mathrm{B}}} \mathrm{f}_{\mathrm{A}}(x, y) \mathrm{d} x \mathrm{~d} y \quad$,

where $r_{\mathrm{B}}$ is the region in the $x, y$ plane associated with response $R_{\mathrm{B}}$.

To account for perceived similarity, Ashby and Perrin (1988) assumed that perceived similarity was proportional to confusability in the absence of response bias. If response bias does not exist, the decision bound is placed where the likelihood ratio

$L(x, y)=\mathrm{f}_{\mathrm{A}}(x, y) / \mathrm{f}_{\mathrm{B}}(x, y)$

equals 1 . Therefore, a measure of the perceived similarity of $S_{\mathrm{A}}$ to $S_{\mathrm{B}}$ in the two-dimensional case is

$s\left(S_{\mathrm{A}}, S_{\mathrm{B}}\right)=k \int_{r_{\mathrm{B}}} \int \mathrm{f}_{\mathrm{A}}(x, y) \mathrm{d} x \mathrm{~d} y$,

where $r_{\mathrm{B}}=[(x, y) \mid L(x, y)<1]$, and $k$ is some positive constant. Thus, the similarity of stimulus $S_{\mathrm{A}}$ to $S_{\mathrm{B}}$ is naturally defined as the proportion of the $S_{\mathrm{A}}$ perceptual distribution falling in the response region assigned to $R_{\mathrm{B}}$ in an unbiased two-choice identification task when $k=1$.

The most familiar version of general recognition theory assumes that the perceptual distributions are multivariate normal. This special case-called the general Gaussian recognition model-is related to the Case I model of Thurstone's Law of Categorical Judgment (Thurstone, 1927; see also Ennis, 1988; Ennis \& Mullen, 1986; Ennis, Palen, \& Mullen, 1988; Hefner, 1958; MacKay, 1989; Torgerson, 1958; Zinnes \& MacKay, 1983). Alternatively, it can be viewed as a multidimensional generalization of signal detection theory (e.g., Graham, Kramer, \& Yager, 1987; Green \& Swets, 1966; Nakatani, 1972; Tanner, 1956; Wandell, 1982).

The parameters of the general Gaussian recognition model include a mean vector and covariance matrix for each stimulus. The diagonal of the covariance matrix contains the variances on each perceptual dimension, and the off-diagonal elements represent the covariances between pairs of dimensions. In this respect, the model is similar to the probabilistic MDS models recently proposed by Ennis et al. (1988) and MacKay (1989).

General recognition theory of similarity can be formulated in two ways, depending on whether the stimulus context is considered (Ashby \& Perrin, 1988). When three or more stimuli are in the ensemble, the decision bounds can be placed in the perceptual space by considering any two stimuli in isolation and ignoring the third stimulus, or by considering the entire ensemble at once. The former is a context-free similarity model and the latter a context-sensitive model.

Ashby and Perrin (1988) showed that traditional MDS models employing a Euclidean distance metric are a special case of the context-free general Gaussian recognition model of similarity. If the following restrictions are placed on the context-free general Gaussian recognition model, it makes similari- 
ty predictions identical to those of the three-mode MDS model (i.e., Equation 1):

1. For each person, all stimuli have the same associated perceptual covariance matrix,

2. All people have the same configuration of perceptual means, and

3. Similarity and dissimilarity are related by

$d\left(S_{\mathrm{A}}, S_{\mathrm{B}}\right)=-2 \Phi^{-1}\left[s\left(S_{\mathrm{A}}, S_{\mathrm{B}}\right)\right]$,

where $\Phi$ is the standard normal cumulative distribution function.

The general Gaussian recognition model that is equivalent to the three-mode model has a perceptual distribution centered at each stimulus point, $\left(u_{j}, v_{j}\right)$, and the covariance matrix associated with every perceptual distribution of person $i$ equals

$\Sigma_{i}=\left[\begin{array}{cc}\frac{1}{w_{u i}^{2} \sin ^{2}\left(\theta_{i}\right)} & \frac{-\cos \left(\theta_{i}\right)}{w_{u i} w_{v i} \sin ^{2}\left(\theta_{i}\right)} \\ \frac{-\cos \left(\theta_{i}\right)}{w_{u i} w_{v i} \sin ^{2}\left(\theta_{i}\right)} & \frac{1}{w_{v i}^{2} \sin ^{2}\left(\theta_{i}\right)}\end{array}\right]$.

Thus, in this version of the model, all perceptual covariance matrices are equal for each person. Because the three-mode model allows each person to have unique values for $\theta, w_{u}$, and $w_{v}$, however, a different covariance matrix may be associated with each person. To find the version of the general Gaussian recognition model that is equivalent to the INDSCAL model, set $\theta=90^{\circ}$. The version that reduces to the Simple Euclidean model is found by setting $\theta=90^{\circ}$ and all weights to 1.0.

In general recognition theory, perceptual independence occurs if the perceptual effects of the dimensions are statistically independent-that is, if the joint probability distribution of the perceptual effects associated with a stimulus is equal to the product of the marginal probability distributions for each dimension (Ashby, 1988; Ashby \& Townsend, 1986). In this case, the effects of the perceptual effects $(x, y)$ of a particular stimulus are statistically independent.

In the special case in which the perceptual distributions are multivariate normal, perceptual independence is equivalent to a zero correlation between the effects of the dimensions. Therefore, when the covariance term of the general Gaussian recognition model is equal to 0 the dimensions are perceived independently, and $\theta$-the angle between dimensions $u$ and $v$ in the corresponding three-mode model-is equal to $90^{\circ}$. Ashby and Townsend (1986) showed that the degree of dependence can be assessed through the relationship $\rho_{x y}=-\cos (\theta)$, where $\rho_{x y}$ is the correlation between dimensions $x$ and $y$. Therefore, because INDSCAL is a special case of the three-mode model in which $\theta=90^{\circ}$, INDSCAL assumes perceptual independence.

The relationships between the various models are summarized in Figure 1. Figure la shows the stimulus representation of four stimuli $\mathrm{A}, \mathrm{B}, \mathrm{C}$, and D, which vary on physical dimensions $X$ and $Y$. Figures $\mathrm{lb}$ through le show four possible perceptual representations of these stimuli according to general recognition theory. Figures $1 \mathrm{f}$ through $\mathrm{li}$ show the four corresponding perceptual representations to the above general recognition theory representations according to MDS. In Figure $1 \mathrm{~b}$, each stimulus has a different associated covariance matrix. For example, when stimulus $\mathrm{A}$ is presented, the perceptual dimensions $x$ and $y$ are perceived independently, but when stimulus B is presented $x$ and $y$ exhibit a negative dependence. In this case, there is no equivalent MDS representation.

In Figure 1c, dimensions $x$ and $y$ exhibit a positive dependence, but the magnitude of this dependence $(\rho)$ does not rely on the stimulus presented. The equivalent MDS representation (Figure $\mathrm{lg}$ ) comes from the three-mode model in which the angle between perceptual dimensions $u$ and $v$ 

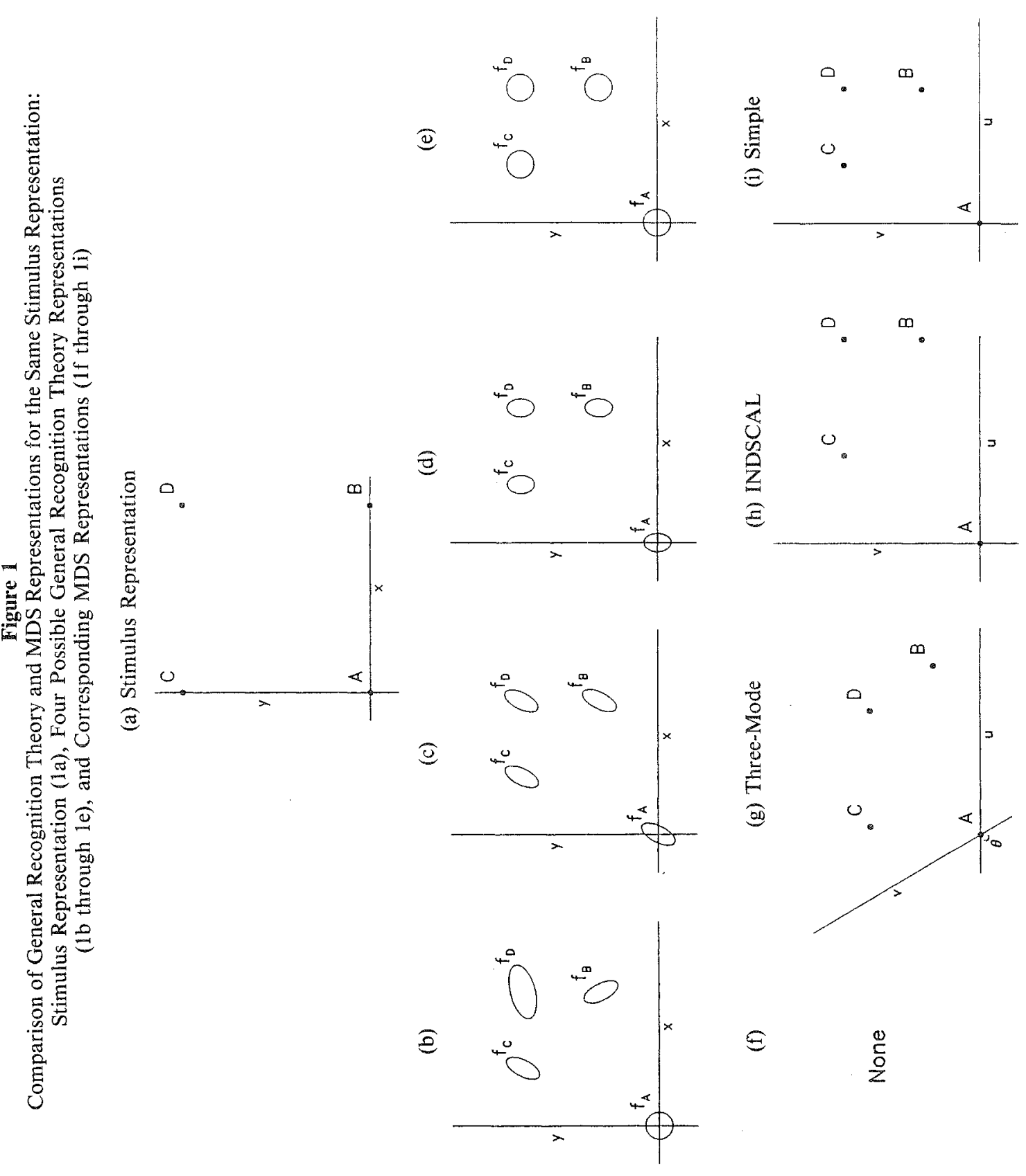

Downloaded from the Digital Conservancy at the University of Minnesota, http://purl.umn.edu/93227. May be reproduced with no cost by students and faculty for academic use. Non-academic reproduction requires payment of royalties through the Copyright Clearance Center, http://www.copyright.com/ 
satisfies $p=-\cos (\theta)$. In Figure 1d, the perceptual dimensions $x$ and $y$ are always perceived independently and all stimuli have the same associated covariance matrix. Note, however, that there is greater variability along dimension $y$ than along dimension $x$. The equivalent MDS representation is generated by the INDSCAL model in which greater weight is placed on dimension $u$. Finally, in Figure le the perceptual dimensions $x$ and $y$ are perceived independently and the variance on the $x$ dimension equals the variance on the $y$ dimension for all stimuli. The equivalent MDS representation (Figure 1i) is generated by the simple Euclidean model in which equal weights are given to the $x$ and $y$ dimensions.

Note that the positions of $\mathrm{B}$ and $\mathrm{C}$ change from the stimulus to the perceptual representations. This illustrates the fact that the stimulus dimensions do not necessarily equal the perceptual dimensions. Furthermore, B and C are not orthogonal in either MDS representation, even though they are orthogonal in the stimulus representation. Even in the INDSCAL model, then, the perceptual dimensions $u$ and $v$ are not determined simply by the image of stimulus pairs that happen to be orthogonal in the stimulus space.

\section{The Dominance Axiom}

The dominance axiom is presented here as a test of raw dissimilarity data in order to determine if the assumption of independent dimensions is satisfied. Tversky and Gati (1982) derived three testable properties of a class of models they referred to as monotone proximity structures. Of particular interest is the property they termed dominance. Consider a set of stimuli, identical except for two physical dimensions. The stimuli can take one of two values on each of these dimensions, with the resulting four stimuli termed $X_{a} Y_{p}, X_{a} Y_{q}, X_{b} Y_{p}$, and $X_{b} Y_{q}$. If $d(i, j)$ represents the dissimilarity between stimuli $i$ and $j$, the dominance axiom states that

$d\left(X_{a} Y_{p}, X_{b} Y_{q}\right)>\max \left[d\left(X_{a} Y_{p}, X_{a} Y_{q}\right), d\left(X_{a} Y_{q}, X_{b} Y_{q}\right)\right]$.

In other words, the dissimilarity of a pair of stimuli differing on two dimensions must exceed the dissimilarities of both pairs of stimuli that differ on only one of these dimensions. Note that Equation 7 only uses three of the four stimuli in the ensemble (stimulus $X_{b} Y_{p}$ is not used). The axiom can be generalized to include all four stimuli:

$d\left(X_{a} Y_{p}, X_{b} Y_{q}\right)>\max \left[d\left(X_{a} Y_{p}, X_{a} Y_{q}\right), d\left(X_{a} Y_{q}, X_{b} Y_{q}\right), d\left(X_{b} Y_{p}, X_{b} Y_{q}\right), d\left(X_{a} Y_{p}, X_{b} Y_{p}\right)\right]$.

Depending on the specific covariance matrix associated with each stimulus distribution, general recognition theory can predict that dominance will be either satisfied or violated. Ashby and Perrin (1988) reported a numerical example in which dominance was violated because of a perceptual dependence. This result raises the possibility that the dominance axiom is closely related to perceptual independence. The following result shows that this sensitivity to perceptual dependence is robust. (The proof of this theorem can be found in the Appendix.)

Theorem: If perceptual independence holds and all perceptual distributions have equal covariance matrices, the context-free general Gaussian recognition model predicts dominance to be satisfied.

This theorem indicates that there are two different ways in which dominance can be violated: (1) if a perceptual dependence occurs, and (2) if not all covariance matrices are equal. Fortunately, dominance is robust with respect to violations of the assumption of equal covariance matrices, even though it is sensitive to violations of independence. For this reason, it makes a powerful test of perceptual independence. 


\section{Sensitivity to Violations of Perceptual Independence}

Numerical investigations (not reported here) revealed that dominance was satisfied with perceptually independent dimensions when the ratio of the largest variance on a given dimension to the smallest variance on the same dimension did not exceed 5.0. Violations of dominance were found only when this ratio was even more extreme. Such extreme violations of the equal covariance matrices condition result in a situation where even the three-mode MDS model is inappropriate (e.g., Figure (b). Unequal covariance matrices would be equivalent to differential weighting of dimensions across stimuli. If an extreme violation of the equal covariance matrices assumption is suspected, violations of the dominance axiom should be interpreted with caution. Because the assumptions of MDS are not met, the dissimilarity data should be interpreted with some nongeometric approach, such as Tversky's (1977) feature-contrast model, or Ashby and Perrin's (1988) general recognition theory.

\section{Simulation Study}

The case was considered in which violations of the equal covariance matrices assumption were not severe; thus some version of MDS was likely to provide an adequate account of the data. The problem of determining the number and type of violations of dominance to be expected from data in which the dimensions are truly independent is discussed and contrasted with results from data collected when the dimensions were dependent.

\section{Generation of Simulated Data}

Three conditions were investigated, each of which began with the same initial configuration of nine stimuli in two dimensions (Figure 2a). The angle between the dimensions $u$ and $v$ varied across the three conditions. For all hypothetical people (simulees) in the first condition, $\theta$ was set at $90^{\circ}$, which corresponded to a correlation of 0 and a perceptual independence in general recognition theory. $\theta$ was set at $75^{\circ}$ in the second condition, which represented a perceptual correlation of .26 in general recognition theory (Figure $2 \mathrm{~b}$ ). $\theta$ was set at $60^{\circ}$ in the third condition, which corresponded to a perceptual correlation of .5 (Figure 2c).

One hundred dissimilarity matrices were generated for each condition. Simulation of each matrix began with the initial configuration and included differential weighting of dimensions, systematic error, and random error. Person weights were generated for each dimension for each of the simulees, and were set at a level that represented moderate individual differences. Weights on dimension $u$ were generated to be uniformly distributed on a $0-1$ interval. Weights on dimension $v$ were then generated over the same interval. To ensure moderate individual differences, each simulee's weight vectorformed by plotting the two weights-was scaled by a constant so that the new length was between .7 and .9. This procedure was followed for each of the three conditions.

Dissimilarities between the nine stimuli were computed for each simulee using the three-mode model (i.e., Equation 1). Note that the three-mode model reduces to the INDSCAL model in the first condition (with an angle of $90^{\circ}$ ). The resulting dissimilarities were then scaled to the 1-to-9 scale typically used in dissimilarity studies. After the scaling, a modification of Girard and Cliff's (1976) error model was used to add the error:

$d^{*}=4.0 \tanh [s(d+e-5.0)]+5.0$,

where $d^{*}$ is the error-perturbed dissimilarity, $s$ is a normally distributed systematic error component with a mean of .25 and standard deviation (SD) of .05, $d$ is the original 1-to-9 scaled dissimilarity, and $e$ is a normally distributed random error component with mean $=0$ and $\mathrm{SD}=.1$. 
Figure 2

Stimulus Configurations for Simulation Data

(a) $\theta=90^{\circ}$

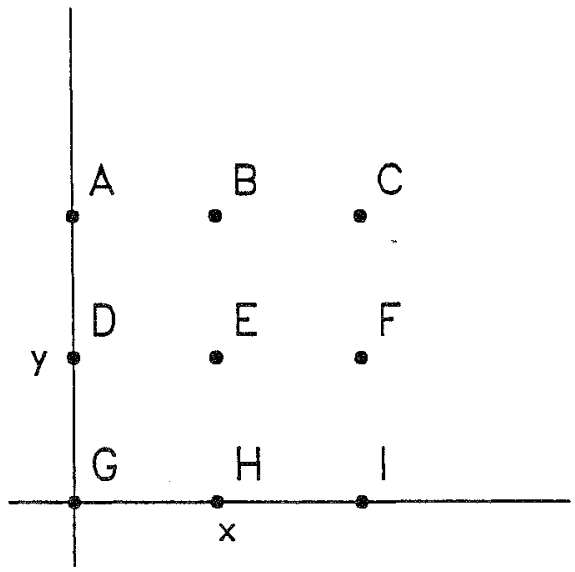

(b) $\theta=75^{\circ}$

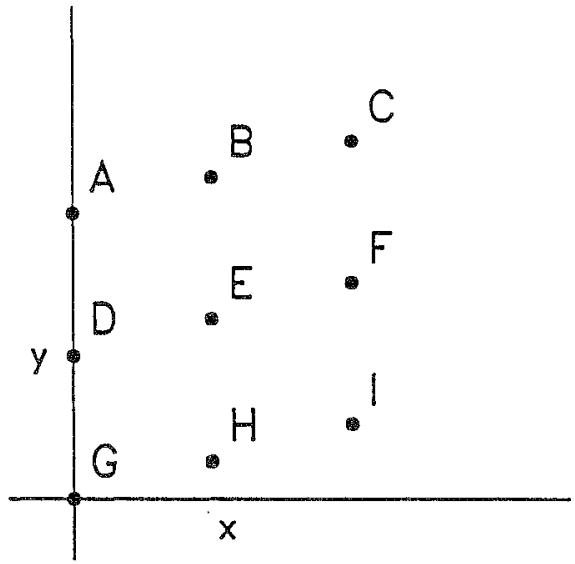

(c) $\theta=60^{\circ}$

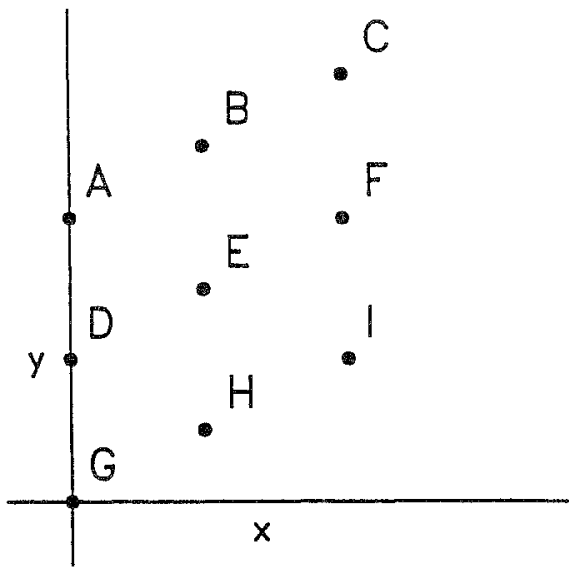


Systematic error represents differences in how people use the response scale. It was set at a level representing moderate individual differences in response style. The systematic error component varied across simulees but was constant within a simulee. Random error was also set at a moderate level, and each simulee had a different random error component for each generated distance. The errorperturbed dissimilarities that resulted from Equation 9 tended to underestimate the true dissimilarities at the upper end of the scale, and to overestimate the true dissimilarities at the lower end of the scale. Each of the 300 error-perturbed dissimilarity matrices was tested for violations of dominance using Equation 8. A violation of the axiom occurred if any of the four one-dimensional dissimilarities exceeded the associated two-dimensional dissimilarity.

\section{Results}

With the configuration of nine stimuli, there were 18 possible tests of the dominance axiom. In other words, there were 18 sets of four stimuli (or quadruples) in which a two-dimensional dissimilarity could be compared to the corresponding one-dimensional dissimilarities.

Condition 1, in which perceptual independence was satisfied, had a mean of 2.41 violations of the dominance axiom out of the 18 possible tests per simulee. A mean of 5.2 violations per simulee was found when $\theta=75^{\circ}$, and a mean of 9.22 violations was found when $\theta=60^{\circ}$. Furthermore, the $60^{\circ}$ condition had a significantly greater number of violations per simulee than the $75^{\circ}$ condition $(t=22.84, d f=99, p<.0001)$, and the $75^{\circ}$ condition had a significantly greater number of violations than the $90^{\circ}$ condition $(t=11.63, d f=99, p<.0001)$.

Testing for a significant number of violations has long been a problem that has plagued axiomatic measurement theory. One solution is to examine the pattern of violations of dominance for each of the three conditions. Note, however, that some quadruples in Figure $2 \mathrm{a}$ are larger than others. The horizontal and vertical distances between each pair of adjacent stimuli is 1 unit (called "twostep quadruples") in 8 of the 18 quadruples (e.g., DBAE, GEDH). Another eight quadruples (threestep quadruples) involve a distance of 2 units on one dimension and 1 unit on the other dimension (e.g., GBAH, GFDI). Finally, two quadruples involve a distance of 2 units on both dimensions. Because

$d(\mathrm{D}, \mathrm{B})-d(\mathrm{D}, \mathrm{A})<d(\mathrm{G}, \mathrm{C})-d(\mathrm{G}, \mathrm{A})$,

violations of dominance that are due to random error alone are more likely in the two-step than the three-step quadruples. However, such violations should be uniformly distributed within all quadruples of the same size.

Conversely, violations of dominance that are due to perceptual dependence are more likely in some quadruples than others, even when quadruple size is controlled. For example, Figure $2 \mathrm{c}$ indicates that frequent violations were predicted for the quadruple DHGE, because $\mathrm{D}$ and $\mathrm{H}$ (which differ on two dimensions) are closer in the space than the corresponding stimulus pairs that differ on only one dimension (DG, HG, DE, and HE). Yet violations should be rare with the quadruple GEDH.

The following testing strategy is suggested. First, test the null hypothesis that the violations are uniformly distributed across the entire set of 18 quadruples. If they are, they must be uniformly distributed across all two-step and three-step quadruples. If the null hypothesis is rejected, then test the same hypothesis separately on the two-step and three-step quadruples.

Table 1 shows the pattern of violations across the entire 18 stimulus quadruples or tests. The number of simulees that had a violation of dominance for a given stimulus quadruple is shown for each test and condition. The first two letters in each quadruple define the two-dimensional dissimilarity that was tested. A Pearson goodness-of-fit test was used to test the null hypothesis that the observed frequency distributions were uniform (Marascuilo \& McSweeney, 1977). The $\chi^{2}$ for this test was not 
Table 1

Number of Violations of the Dominance Axiom Per Stimulus Quadruple for Simulated Data

\begin{tabular}{lccr}
\hline \hline Quadruple & $\theta=90^{\circ}$ & $\theta=75^{\circ}$ & $\theta=60^{\circ}$ \\
\hline 1 GEDH & 15 & 9 & 4 \\
2 GBAH & 15 & 6 & 4 \\
3 GFDI & 9 & 9 & 3 \\
4 GCAI & 8 & 4 & 3 \\
5 DHGE & 18 & 44 & 96 \\
6 DBAE & 14 & 6 & 4 \\
7 DIGF & 11 & 60 & 98 \\
8 DCAF & 11 & 5 & 5 \\
9 AHGB & 19 & 58 & 98 \\
10 AEDB & 12 & 53 & 99 \\
11 AIGC & 13 & 42 & 99 \\
12 AFDC & 11 & 58 & 99 \\
13 HFEI & 14 & 3 & 4 \\
14 HCBI & 12 & 6 & 3 \\
15 EIHF & 16 & 38 & 99 \\
16 ECBF & 11 & 11 & 7 \\
17 BIHC & 17 & 67 & 99 \\
18 BFEC & 15 & 41 & 98 \\
\hline
\end{tabular}

significant in the first condition $\left(\chi^{2}=11.67, d f=17\right)$; thus, the violations found when $\theta=90^{\circ}$ follow no consistent pattern, which is predicted by the general recognition theory account of dominance.

The pattern of violations in the other two conditions clearly were not uniform. The $\chi^{2} \mathrm{~s}$ for the $75^{\circ}$ and $60^{\circ}$ conditions were 341.64 and 780.35 , respectively ( $d f=17, p<.001$ for both tests). Because the null hypothesis was rejected using all 18 quadruples, the two-step and three-step quadruples were tested separately for each condition in order to test whether the violations were uniform within the two-step and three-step quadruples. The $\chi^{2}$ s were significant $(d f=7, p<.001)$ in all cases $\left(\chi^{2}=111.76\right.$ and $\chi^{2}=176.95$ for the two-step and three-step quadruples, respectively, in the $75^{\circ}$ condition; $\chi^{2}=291.56$ and $\chi^{2}=351.27$ for the two- and three-step quadruples, respectively, in the $60^{\circ}$ condition). Therefore, the hypothesis of a uniform distribution of violations of the dominance axiom in the $75^{\circ}$ and $60^{\circ}$ conditions was not supported.

The perceptual dependencies actually have the effect of making violations of dominance more likely for some quadruples and less likely for others. Figure $2 \mathrm{c}$ shows that the observed pattern is predicted by general recognition theory and by the three-mode model. For example, frequent violations of dominance were correctly predicted for the quadruple DHGE, because D and H (which differ on two dimensions) are closer in the space than DG, HG, DE, and HE (which differ on only one dimension). Conversely, the quadruple GEDH was correctly predicted almost never to violate dominance. (Both these quadruples are two-step quadruples.) Therefore, both the number and the pattern of violations are important in determining whether the dimensions are perceived independently.

The pattern of violations can be examined further to see if it is consistent with a positive or negative dependency. A positive dependency represents the case in which the perceived position on one dimension increases as the position on the other dimension increases. A negative dependency represents the case in which the perceived position on one dimension decreases as the position on the other dimension increases. For example, violations would be expected in DHGE but not GEDH under positive dependency. 
This is exactly the pattern found in the $75^{\circ}$ and $60^{\circ}$ conditions in which positive dependencies exist. Quadruples $5,7,9,10,11,12,15,17$, and 18 would be expected to contain violations of dominance, whereas the other quadruples would not.

The dominance axiom should be able to detect any departure from $90^{\circ}$. However, the smaller the departure is, the harder it is to detect. As with any test, this is a function of power; more people are needed to detect smaller departures.

\section{Empirical Application}

To examine the generality of the simulation results, data on the size-weight illusion collected by Dunn (1976) were tested for violations of the dominance axiom. The size-weight illusion is a case in which perceptual dependencies often arise, and thus general recognition theory predicts a consistent pattern of violations.

\section{Dunn's Experiment}

In Dunn (1976; Dunn \& Harshman, 1982), people rated the dissimilarity of pairs of stimuli varying in size and weight. The stimuli were plastic blocks painted flat black with metal lifting rings on top. The blocks were cubes in which the length of a side was 50,65 , or $88 \mathrm{~mm}$. The mass of the blocks was 100,150 , or $225 \mathrm{gm}$. The two dimensions were factorially combined, which created nine different stimuli. The configuration of these nine stimuli is the same as the configuration used in the simulation study.

Nineteen people made dissimilarity ratings on a scale ranging from 0 (exactly alike) to 9 (very dissimilar). Seventy-two different pairs were judged by each person, and identical pairs were not presented. The 72 pairs were presented twice to each person, creating a total of 144 judgments per person. Dunn computed the mean of the four dissimilarity ratings for each pair of stimuli generated by every person, and thus produced a symmetric $9 \times 9$ dissimilarity matrix for each person. He found good fit to his data for both the INDSCAL model and the three-mode model.

\section{Results of the Reanalysis}

With the nine stimuli of Dunn's (1976) experiment, 18 tests of dominance were possible. Table 2 shows the number of violations for each quadruple when dominance was tested on the mean dissimilarity data for each person. A weaker test of dominance is also presented in Table 2 . In this case, the strict inequality in Equation 8 was changed to $\leq$. Because the data were collected using a 0-to-9 scale, it can be argued that some of the ties in the judgments do not represent strict equality in perceived dissimilarity, but rather are the result of the restrictive nature of the rating scale. These ties can represent perceived dissimilarities that are less than, greater than, or equal to other judgments of the same value. Thus, the weaker test of dominance can be considered an upper bound on the number of violations that might occur with a larger rating scale.

Some quadruples in Table 2 were more frequently associated with violations of dominance than others. These quadruples also showed the greatest increase in the number of violations for the weaker test. Specifically, it appears that dominance was not violated in all quadruples, but was frequently violated in quadruples $5,9,10,15,17$, and 18 . There were at least nine violations out of the possible 19 upper-bound tests in these quadruples, whereas there were seven or fewer violations in the other quadruples. Violations in these quadruples would be expected when the dependency is positive, as in the case of the size-weight illusion.

The pattern of violations also closely resembles the pattern found in the simulation study, with a few exceptions discussed below. The Pearson goodness-of-fit test performed on the strict dom- 
Table 2

Number of Violations of the Dominance Axiom for the Size-Weight Illusion Data $(\mathrm{A}=225 \mathrm{gm}, 50 \mathrm{~mm} ; \mathrm{B}=150 \mathrm{gm}, 50 \mathrm{~mm}$; $\mathrm{C}=100 \mathrm{gm}, 50 \mathrm{~mm} ; \mathrm{D}=225 \mathrm{gm}, 65 \mathrm{~mm}$; $\mathrm{E}=150 \mathrm{gm}, 65 \mathrm{~mm} ; \mathrm{F}=100 \mathrm{gm}, 65 \mathrm{~mm}$; $\mathrm{G}=225 \mathrm{gm}, 88 \mathrm{~mm} ; \mathrm{H}=150 \mathrm{gm}, 88 \mathrm{~mm}$; $\mathrm{I}=100 \mathrm{gm}, 88 \mathrm{~mm})$

\begin{tabular}{crc}
\hline \hline Quadruple & $\begin{array}{c}\text { Strict } \\
\text { Test }\end{array}$ & $\begin{array}{c}\text { Upper-Bound } \\
\text { Test }\end{array}$ \\
\hline 1 GEDH & 5 & 6 \\
2 GBAH & 2 & 3 \\
3 GFDI & 2 & 3 \\
4 GCAI & 1 & 2 \\
5 DHGE & 11 & 12 \\
6 DBAE & 3 & 3 \\
7 DIGF & 3 & 4 \\
8 DCAF & 1 & 2 \\
9 AHGB & 12 & 14 \\
10 AEDB & 5 & 9 \\
11 AIGC & 2 & 3 \\
12 AFDC & 3 & 3 \\
13 HFEI & 6 & 7 \\
14 HCBI & 1 & 5 \\
15 EIHF & 9 & 10 \\
16 ECBF & 5 & 7 \\
17 BIHC & 10 & 13 \\
18 BFEC & 10 & 14 \\
\hline
\end{tabular}

inance data yielded $\chi^{2}=47.09(p<.001, d f=17)$. This test was also performed on the two- and three-step quadruples for the strict dominance data. The hypothesis that the three-step quadruples follow a uniform distribution was rejected $\left(\chi^{2}=30.0, d f=7, p<.001\right)$. However, the test was not significant with the two-step quadruples, which are more sensitive to error. Because the distribution was not uniform within the three-step quadruples and dominance is less likely to be violated in these larger quadruples by random error alone, it can be concluded that the overall pattern of violations does not follow a uniform distribution. Therefore, the dimensions of size and weight were not perceived independently and the appropriate model for the data is the three-mode model.

When the pattern of violations found in the size-weight data were compared with the simulation results, the quadruples 7,11 , and 12 did not exhibit frequent violations in the size-weight data, even though they did in the simulation data. These three quadruples compared stimuli that differed across two levels of weight $(225 \mathrm{gm}$ and $100 \mathrm{gm})$. The quadruples that did exhibit the expected pattern of violations differed by only one level of weight ( $225 \mathrm{gm}$ versus $150 \mathrm{gm}$, or $150 \mathrm{gm}$ versus $100 \mathrm{gm}$ ). As the differences in weight increased, the effect of the size-weight illusion appeared to decrease. The test of dominance allows discovery of this differential effect of the illusion, whereas solely examining the scaling solution does not.

\section{Summary and Discussion}

The results of both the simulation and empirical studies suggest that dominance is a powerful 
test of perceptual independence for dissimilarity data. The number of violations of the dominance axiom increases with the degree of perceptual dependence of the dimensions. However, not only the number of violations is important, but also the pattern of violations across the separate tests of dominance. Perceptually dependent dimensions have the effect of making violations of dominance more likely in some quadruples of stimuli in the perceptual space, and less likely in other quadruples. This pattern is further determined by whether the dependency is positive or negative. A clear, consistent pattern of violations is strong evidence that the dimensions are not perceived independently.

It was shown that the three-mode model is a special case of the general recognition theory that allows for perceptually dependent dimensions. Therefore, it can predict violations of the dominance axiom. The INDSCAL model, which assumes independent dimensions, restricts the covariance term of general recognition theory to be 0 and cannot account for violations of the dominance axiom. Simply finding a good fit of the INDSCAL model with no negative person weights does not guarantee that the dimensions are perceived independently.

Dunn (1976) found good fit of the INDSCAL model to his data and no negative weights, yet the size-weight illusion is a well-known case of perceptually dependent dimensions. A consistent pattern of violations arose when his data were tested for violations of dominance. This suggests that the dimensions were not perceived independently, and that the dimensions recovered by the INDSCAL solution may not represent the true perceptual dimensions. Thus, the three-mode model representation is more appropriate than the INDSCAL solution for his data. The pattern of violations in Dunn's data also suggests that the illusion decreases as the magnitude of weight differences increases, which would not be discovered by simply examining either the INDSCAL or three-mode solutions.

The use of dominance to test for perceptual independence provides important information about how people process stimulus dimensions. Because there may exist an equivalent INDSCAL model to a three-mode model and both solutions may lead to good fit, however, a separate test of independence on dissimilarity data is necessary to determine which MDS model is appropriate. A clear, consistent pattern of violations of the dominance axiom is strong evidence for perceptually dependent dimensions, and the three-mode model will thus lead to a more appropriate interpretation of the perceptual space. In such cases, an INDSCAL solution may indicate good fit to the data, but the dimensions recovered by INDSCAL will not correspond closely to the perceptually dependent dimensions employed by the people (MacCallum, 1976a). Few violations and a random pattern of violations of dominance are evidence that the INDSCAL model's assumption of independence is satisfied, and therefore that a good-fitting INDSCAL solution should lead to the correct interpretation of the data.

\section{Appendix}

Proof: For convenience and without loss of generality, assume the $X_{a} Y_{p}$ mean is $(0,0)$, the $X_{a} Y_{q}$ mean is $\left(0, \mu_{y}\right)$, the $X_{b} Y_{p}$ mean is $\left(\mu_{x}, 0\right)$, and the $X_{b} Y_{q}$ mean is $\left(\mu_{x}, \mu_{y}\right)$. Suppose the variance on each dimension is $\sigma_{x}^{2}$ and $\sigma_{y}^{2}$. As long as similarity and dissimilarity are inversely related, the dominance axiom (i.e., Equation 7) can be written in terms of similarities as

$s\left(X_{a} Y_{p}, X_{b} Y_{q}\right)<\min \left[s\left(X_{a} Y_{p}, X_{a} Y_{q}\right), s\left(X_{a} Y_{q}, X_{b} Y_{q}\right)\right]$.

Equation 4 is used to compute these similarities in the general Gaussian recognition model. Ashby and Perrin (1988) show that under the conditions of the theorem and $k$ arbitrarily set to 1.0, the Equation 4 integral reduces (to Morrison, 1976, p. 235)

$s\left(S_{i}, S_{j}\right)=\Phi\left(-\frac{1}{2 d_{i j}}\right)$, 
where $\Phi$ is the standard normal cumulative distribution function and $d_{i j}$ is the Mahalanobis distance between the means of stimuli $S_{i}$ and $S_{j}$. Therefore dominance is satisfied if

$\Phi\left(-\frac{1}{2 d_{a p, b q}}\right)<\min \left[\Phi\left(-\frac{1}{2 d_{a p, a q}}\right), \Phi\left(-\frac{1}{2 d_{a q, b q}}\right)\right]$,

or equivalently if

$d_{a p, b q}>\max \left(d_{a p, a q}, d_{a q, b q}\right)$.

Now

$d_{i j}=\left(\mu_{i}-\mu_{j}\right)^{\mathrm{T}} \mathbf{\Sigma}^{-1}\left(\mu_{i}-\mu_{j}\right)$.

Substituting specific parameter values and simplifying reveals that dominance holds if

$\frac{\mu_{x}^{2}}{\sigma_{x}^{2}}+\frac{\mu_{y}^{2}}{\sigma_{y}^{2}} \max \left(\frac{\mu_{x}^{2}}{\sigma_{x}^{2}}, \frac{\mu_{y}^{2}}{\sigma_{y}^{2}}\right)$,

which is true for all values of $\mu_{x}, \mu_{y}, \sigma_{x}^{2}$, and $\sigma_{y}^{2}$. The theorem can be shown to hold for the more general Equation 8 version of dominance in an analogous fashion.

\section{References}

Ashby, F. G. (1988). Estimating the parameters of multidimensional signal detection theory from simultaneous ratings on separate stimulus components. Perception and Psychophysics, 44, 195-204.

Ashby, F. G., \& Gott, R. E. (1988). Decision rules in the perception and categorization of multidimensional stimuli. Journal of Experimental Psychology: Learning, Memory, and Cognition, 14, 33-53.

Ashby, F. G., \& Maddox, W. T. (1990). Integrating information from separable psychological dimensions. Joumal of Experimental Psychology: Human Perception and Performance, 16, 598-612.

Ashby, F. G., \& Perrin, N. A. (1988). Toward a unified theory of similarity and recognition. Psycho logical Review, 95, 124-150.

Ashby, F. G., \& Townsend, J. T. (1986). Varieties of perceptual independence. Psychological Review, 93, 154-179.

Bloxom, B. (1978). Constrained multidimensional scaling in $N$ spaces. Psychometrika, 43, 283-319.

Carroll, J. D., \& Chang, J. J. (1970). Analysis of individual differences in multidimensional scaling via an $N$-way generalization of "Eckart-Young" decomposition. Psychometrika, 35, 283-319.

Carroll, J. D., \& Chang, J. J. (1972). IDIOSCAL (individual differences in orientation scaling): A generalization of INDSCAL allowing idiosyncratic reference systems. Paper presented at the Psychometric Society meeting, Princeton NJ, U.S.A.

Carroll, J. D., \& Wish, M. (1974). Models and methods for three-way multidimensional scaling. In $\mathrm{D}$. $\mathrm{H}$. Krantz, R. C. Atkinson, R. D. Luce, \& P. Suppes (Eds.), Measurement, psychophysics, and neural information processing, II (pp. 57-105). New York: Freeman.

Dunn, T. R. (1976). Modelling the core matrix in three mode multidimensional scaling: An example using the size-weight illusion. Unpublished doctoral dissertation, University of Illinois.

Dunn, T. R., \& Harshman, R. A. (1982). A multidimensional scaling model for the size-weight illusion. Psychometrika, 47, 25-45.

Ennis, D. M. (1988). Confusable and discriminable stimuli: Comments on Nosofsky (1986) and Shepard (1986). Journal of Experimental Psychology: General, 117, 408-411.

Ennis, D. M., \& Mullen, K. (1986). A multivariate model for discrimination methods. Journal of Mathematical Psychology, 30, 206-219.

Ennis, D. M., Palen, J. J., \& Mullen, K. (1988). A multidimensional stochastic theory of similarity. Journal of Mathematical Psychology, 32, 449-465.

Girard, B. A., \& Cliff, N. (1976). A Monte Carlo evaluation of interactive MDS. Psychometrika, 41, 43-64.

Graham, N., Kramer, P., \& Yager, D. (1987). Signal detection models for multidimensional stimuli: Probability distributions and combination rules. Journal of Mathematical Psychology, 31, 192-206.

Green, D. M., \& Swets, J. A. (1966). Signal detection theory and psychophysics. New York: Wiley. 
Hefner, R. A. (1958). Extensions of the law of comparative judgment to discriminable and multidimensional stimuli. Unpublished doctoral dissertation, University of Michigan.

Horan, C. B. (1969). Multidimensional scaling: Combining observations when individuals have different perceptual structures. Psychometrika, 34, 139-165.

MacCallum, R. C. (1976a). Effects on INDSCAL of non-orthogonal perceptions of object space dimensions. Psychometrika, 41, 177-188.

MacCallum, R. C. (1976b). Transformation of a threemode multidimensional scaling solution to INDSCAL form. Psychometrika, 4I, 385-400.

MacKay, D. (1989). Probabilistic multidimensional scaling: An anisotropic model for distance judgement. Journal of Mathematical Psychology, 33, 187-205.

Marascuilo, L. A., \& McSweeney, M. (1977). Nonparametric and distribution-free methods for the social sciences. Monterey CA: Brooks/Cole.

McGee, V. C. (1968). Multidimensional scaling of $n$ sets of similarity measures: A nonmetric individual differences approach. Multivariate Behavioral Research, 3, 233-248.

Morrison, D. F. (1976). Multivariate statistical methods (2nd ed.). New York: McGraw-Hill.

Nakatani, L. H. (1972). Confusion-choice model for multidimensional psychophysics. Journal of Mathematical Psychology, 9, 104-127.

Perrin, N. A. (1986). The general recognition theory of preference: A new theory of choice. Unpublished doctoral dissertation, Ohio State University.

Tanner, W. P. (1956). Theory of recognition. Journal of the Acoustical Society of America, 28, 882-888.

Thurstone, L. L. (1927). A law of comparative judg- ment. Psychological Review, 34, 273-286.

Torgerson, W. S. (1958). Theory and methods of scaling. New York: Wiley.

Tucker, L. R. (1972). Relations between multidimensional scaling and three-mode factor analysis. Psychometrika, 37, 3-28.

Tversky, A. (1977). Features of similarity. Psychological Review, 84, 327-352.

Tversky, A., \& Gati, I. (1982). Similarity, separability, and the triangle inequality. Psychological Review, 89, 123-154.

Wandell, B. A. (1982). Measurement of small color differences. Psychological Review, 89, 281-302.

Young, F. W., \& Lewyckyj, R. (1979). Principal directions scaling: A new individual differences model. Paper presented at the Psychometric Society meeting, Monterey CA, U.S.A.

Zinnes, J. L., \& MacKay, D. B. (1983). Probabilistic multidimensional scaling: Complete and incomplete data. Psychometrika, 48, 27-48.

\section{Acknowledgments}

Both authors thank Robert C. MacCallum for his helpful comments on an earlier draft of this paper. Thanks also to Terrence R. Dunn for providing the size-weight illusion data and for his time in explaining his previous research in detail.

\section{Author's Address}

Send requests for reprints or further information to Nancy A. Perrin, Department of Psychology, Portland State University, P.O. Box 751, Portland OR 97207, U.S.A. 